\title{
Poster: Dynamic Time Transformation for Interpreting Clusters of Trajectories with Space-Time Cube
}

\author{
Gennady Andrienko, Natalia Andrienko \\ University of Bonn and Fraunhofer Institute IAIS
}

\begin{abstract}
We propose a set of techniques that support visual interpretation of trajectory clusters by transforming absolute time references into relative positions within temporal cycles or with respect to the starting and/or ending times of the trajectories. We demonstrate the work of the approach on a real data set about individual movement over one year.
\end{abstract}

\section{INTRODUCTION}

Progress in positioning technology and availability of tracking devices created opportunities for collecting large amounts of movement data. Such data may be very useful for optimizing traffic, improving commercial and public services and quality of life in general. One of the major approaches to overcome the problem of large data size is clustering, or grouping trajectories by similarity. In the recent decade, numerous trajectory clustering methods were developed in the data mining [7] and visual analytics [2][8][9] communities.

Visualization is essential for interpreting clusters. The spatial aspect of trajectory clusters can be explored using the method suggested in [3], which represents clusters as flows (Figure 1). Space-time cube (STC) [4] is often used for exploring temporal and spatio-temporal aspects of trajectories. This visualization method has been advanced in several studies [6][1][5].

Exploration of a large number of trajectories with a STC may be hindered by visual clutter (Figure 2). Grouping of spatially similar trajectories and transformations of their temporal references can reduce this problem. Commonalities and differences among temporal and dynamic characteristics of spatially similar trajectories can be revealed. We demonstrate this using a set of about 365 car trajectories of one person recorded during one year. Density-based clustering with the "route similarity" distance function [2][8] detected 9 major clusters of spatially similar trajectories (Figure 1); 123 trajectories were not included in clusters. The straightforward application of the STC (Figure 2) is not helpful due to the visual clutter.

\section{INTERACTIVITY OF SPACE-TIME CUBE.}

Previous works suggest the following basic interactivity of STC:

- $\quad$ change of the viewpoint;

- $\quad$ selection of spatio-temporal objects to be displayed;

- $\quad$ access to objects by pointing and dragging;

- $\quad$ zooming in the spatial (horizontal dimension) and temporal (vertical dimension) extents;

- $\quad$ animation of the content of STC;

- moveable plane for additional temporal reference.

We propose to extend this set of techniques by various transformations of time in respect to the lifelines of the trajectories. We have designed two classes of transformations:

IEEE Symposium on Visual Analytics Science and Technology October 24 - 29, Salt Lake City, Utah, USA

978-1-4244-9486-6/10/\$26.00 @2010 IEEE
1. Transformations that reflect the cyclic nature of time Depending on the data and application, it may be useful to project trajectories to a single year / season / month / week / day / hour etc.

2. Transformations with respect to the individual lifelines of trajectories. Thus, it may be useful to adjust the times of spatially similar trajectories by aligning their start and/or end times.

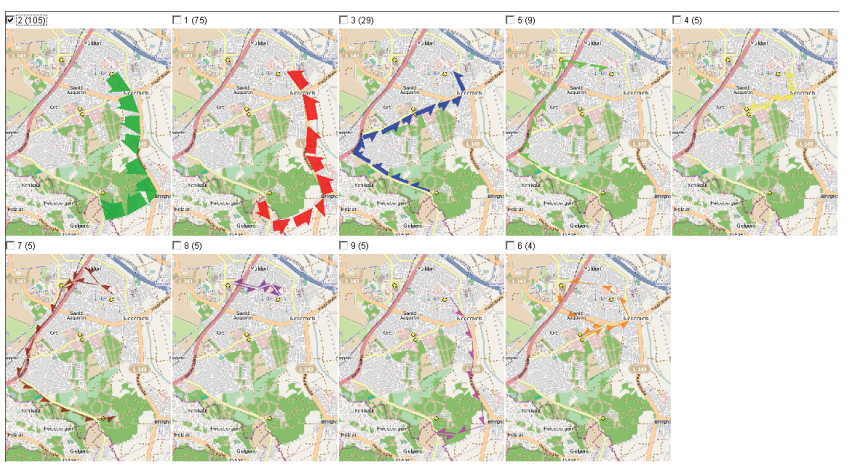

Figure 1. Spatial profiles of 9 clusters.

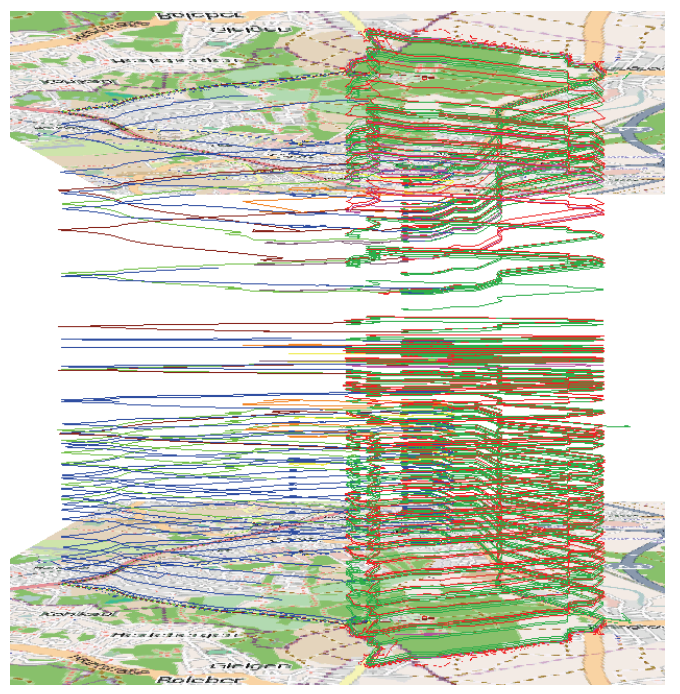

Figure 2. 242 trajectories in a space-time cube. Different colors represent cluster membership.

Figure 3 (top) shows a STC with trajectories adjusted by the weekly cycle. The time axis is oriented upward. The large red, green, and blue clusters occur on the working days. The yellow and orange clusters occur on Saturday. Sunday has only out-ofcluster trajectories, which are not displayed. Figure 3 (bottom) represents the same clusters of trajectories adjusted by the daily cycle. It is easy to see that the green cluster occurs mostly in the morning, red and blue in the evening, and yellow and orange in the middle of the day. 
In Figure 4, we have aligned the starting times of the trajectories. On the left the starting 60 minutes of all clusters are shown. One cluster is shown separately on the right. Most of the trajectories of the green and red clusters have short duration. Few trajectories of these clusters have intermediate stops (indicated by vertical lines), which increase their durations. Almost all trajectories from the blue cluster have stops of variable duration in the same place.

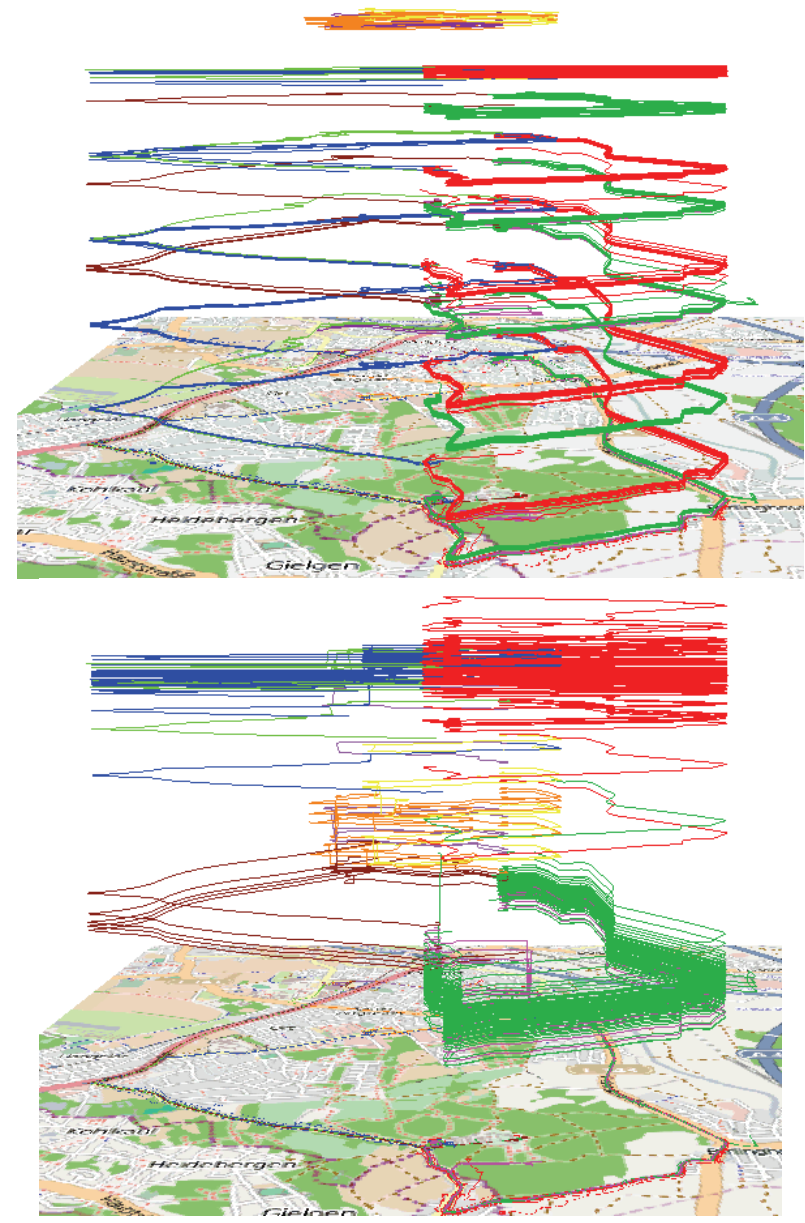

Figure 3. Weekly (top) and daily (bottom) arrangements

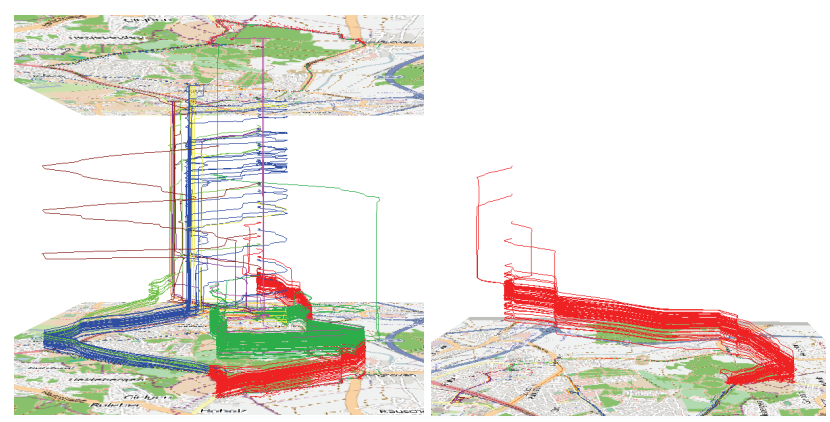

Figure 4. Trajectories are aligned by their starts

It is also possible to align the ending times of the trajectories. Figure 5 presents the STC with the end-aligned trajectories seen from two different viewpoints. Only the last 30 minutes of the trajectories are displayed. Such representation complements the previous technique for interpreting the dynamics within clusters.

The transformation by aligning both the starts and the ends of trajectories is useful for comparing the dynamics of trajectories belonging to the same cluster. Figure 6 shows the STC with a single cluster of trajectories. It can be seen that only a few cluster members differ in their dynamics characteristics from the majority of the trajectories.

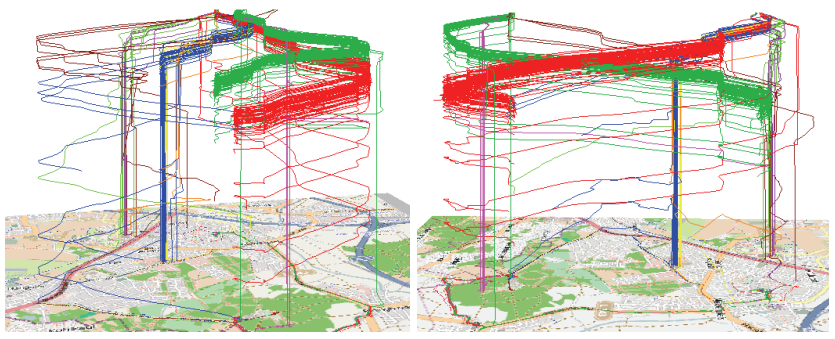

Figure 5. Trajectories are aligned by their ends.

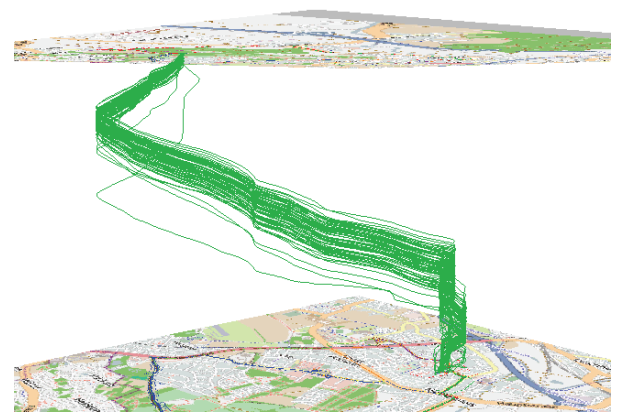

Figure 6. Trajectories are aligned by their starts and ends.

The time transformation techniques are implemented in the Geospatial Visual Analytics toolkit [2] that integrates visual and computational analysis methods.

\section{ACKNOWLEDGEMENT}

This work is supported by DFG within the priority research program on visual analytics (SPP 1335).

\section{REFERENCES}

[1] Andrienko, N., Andrienko, G., and Gatalsky, P. Visual Data Exploration using Space-Time Cube. In Proceedings of the 21st International Cartographic Conference, Durban, 10 - 16 August 2003. International Cartographic Association, pp. 1981-1983.

[2] Andrienko, G., Andrienko, N., and Wrobel, S.: Visual analytics tools for analysis of movement data, ACM SIGKDD Explorations, 9(2), 2007, 38-46

[3] Andrienko, N., and Andrienko, G. Spatial Generalization and Aggregation of Massive Movement Data. IEEE Transactions on Visualization and Computer Graphics (TVCG), 2010 (accepted), http://doi.ieeecomputersociety.org/10.1109/TVCG.2010.44

[4] Hägerstrand, T. What about people in regional science? Papers, Regional Science Association, 24, 7-21. 1970

[5] Kapler, T. and Wright, W. 2005. GeoTime information visualization, Information Visualization, 4(2), 136-146

[6] Kraak, M.-J. 2003. The space-time cube revisited from a geovisualization perspective, in: Proc. of the 21st International Cartographic Conference, Durban, 2003, pp.1988-1995

[7] Li, Y., Han, J., and Yang, J. Clustering moving objects. In Proceedings ACM KDD 2004, 617-622

[8] Rinzivillo, S., Pedreschi, D., Nanni, M., Giannotti, F., Andrienko, N., and Andrienko, G.: Visually-driven analysis of movement data by progressive clustering, Information Visualization, 7(3/4), 2008, 225-239

[9] Schreck, T., Bernard, J., Tekusova, T., and Kohlhammer, J. Visual cluster analysis in trajectory data using editable Kohonen maps. In Proc. IEEE VAST 2008, 2008, 3-10 